\title{
Thermography-based blood perfusion imaging in hands: spectral amplification and time shift
}

\author{
by A.A. Sagaidachnyi, D.A. Usanov, A.V. Skripal, A.V. Fomin
}

Dept. of Nano - and Biomedical Technology, Saratov State University, Saratov, Russia, andsag@yandex.ru

\begin{abstract}
The reasons for differences in the waveform and the delay between the blood flow and temperature oscillations at the skin surface are far from being fully understood. In this study, we determine the relationships between spectral components of the blood flow and temperature oscillations in fingertips, and we ascertain the frequency dependences of amplitude attenuation and delay time for the temperature oscillations. The time-frequency analysis of oscillations was based on the Morlet wavelet transform. The frequency dependencies of delay time and amplitude attenuation in temperature compared with blood flow oscillations have been determined in endothelial $(0.005-0.02 \mathrm{~Hz})$ and neurogenic $(0.02-0.05 \mathrm{~Hz})$ frequency bands using the wavelet spectra. Application of established frequency dependencies in each point of thermograms allowed us to visualize of skin blood perfusion in hands. The method can be considered as the procedure of converting skin temperature into photoplethysmographic signal, and vice versa.
\end{abstract}

\section{Introduction}

Dynamic IR-thermography has great opportunities in a non-contact monitoring of skin engraftment and researches of burn injuries or chilblains [1,2], herewith the temperature distribution defined mainly by skin blood perfusion. At the same time, a number of previous studies have been demonstrated differences in a waveform of temperature and blood flow signals [3-7]. In addition, there is the delay time between simultaneously registered blood flow and temperature signals [3, 4]. Finding interrelation between temperature and skin blood flow, therefore, is the fundamental problem of medical thermal imaging.

The aim of the present study was determination of relationship between temperature and blood flow signals in a spectral domain and application the relationship for blood perfusion imaging in hands with high spatial and temporal resolution.

To achieve the objective we used the envelope of photoplethysmography signal from finger as a characteristic of peripheral blood flow oscillations and thermal IR-camera for skin temperature measurements of hands. Than, we applied wavelet transform to the signals for estimation of spectral components of the temperature and blood flow oscillations. The spectral components have been compared at each frequency so as to plot frequency dependencies of amplitude attenuation and time shift of the temperature.

\section{Infrared imaging and photoplethysmography measurements}

The study included ten normal healthy subjects who were not on medication and who had no history of cardiovascular diseases. All subjects were non-smokers. Their mean \pm standard deviation age was $30 \pm 5$ years. Simultaneous measurements of skin temperature and blood flow of the fingertips were performed under comfortable conditions at close to the constant room temperature $23 \pm 0.2^{\circ} \mathrm{C}$. The subjects were wearing light clothing.

Photoplethysmography-assessed blood flow and temperature signals were measured during 20 minutes at rest. Photoplethysmographic signal was measured by reflectance sensor KL-72001(Taiwan). Temperature of the skin was measured using the thermal imaging camera ThermaCam SC 3000 Flir Systems (Sweden) operating in the spectral range 8-9 $\mu \mathrm{m}$ with temperature sensitivity $0.02{ }^{\circ} \mathrm{C}$. Room temperature was controlled by Davis Vantage Pro 2 Weather Station (USA). Measurements were carried out in a sitting position, hands placed on the surface with low heat capacity (foam plastic). The time adaptation of each subject to the environment was 10-15 minutes. The index finger was placed at the top of the photoplethysmographic sensor. Average temperature within the rectangular area was registered at the lateral side of the distal finger phalanx. The sampling rate of the temperature measurements was $2 \mathrm{~Hz}$, and for the photoplethysmography measurements $-50 \mathrm{~Hz}$. The envelope of the peaks of photoplethysmographic pulse waves was used as a signal characterizing the blood flow changes.

\section{Skin temperature oscillations as thermal waves generated by blood flow oscillations}

To analyze relationship between the temperature and skin blood flow oscillations in a spectral domain we introduced the following theoretical assumptions:

a) Blood flow oscillations are the main cause of variation in skin temperature;

b) Blood flow oscillations are the source of thermal waves traveling from some depth to the surface, and detected by IR-camera as skin temperature oscillations;

c) The thermal waves under consideration characterized by amplitude attenuation and dispersion. 
Thus, definition of frequency dependencies of attenuation and time shift of the temperature signal relative to blood flow signal is necessary for determination of the quantitative relationship between the physiological signals. The frequency dependencies of attenuation and time shift were determined in our recent study [8]. Spectral decomposition of the temperature and photoplethysmography-measured blood flow performed by means of Morlet wavelet transform. As a result the frequency dependencies of the amplitude attenuation (figure $1 \mathrm{a}$ ) and the time shift (figure $1 \mathrm{~b}$ ) of temperature oscillations relative to the blood flow oscillations have been determined (see the original paper [8] for details).

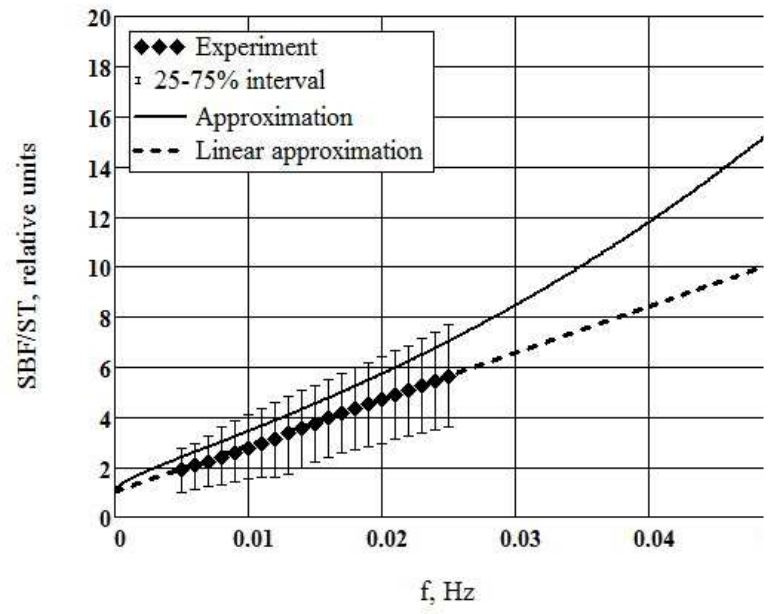

(a)

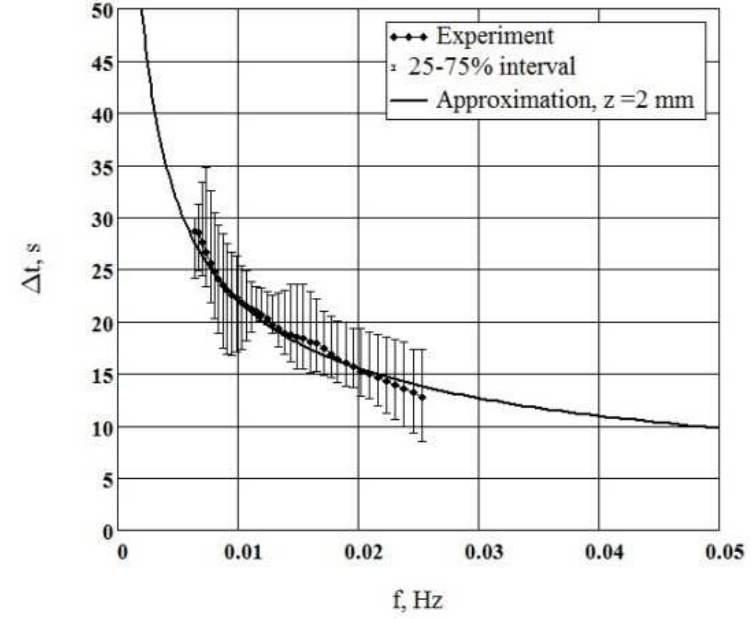

(b)

Fig.1. Frequency dependencies of the amplitude attenuation (a) and the time delay (b) of temperature wavelet spectral components compared with blood flow components (for the group of ten healthy volunteers) [8]

Results presented in figure 1 allowed us to write the expression relating the spectral components of the temperature $S_{T}(f, t)$ and blood flow oscillations $S_{B F}(f, t)$ as

$$
\begin{gathered}
S_{\mathrm{BF}}(f, t)=C_{\mathrm{AMP}}(f, z) \cdot S_{\mathrm{T}}\left(f, t+\Delta t_{\mathrm{PHASE}}(f, z)\right), \\
C_{\mathrm{AMP}}(f, z)=\exp \left(z \cdot \sqrt{\frac{\pi \cdot c \cdot \rho}{\lambda} \cdot f}\right), \\
\Delta t_{\mathrm{PHASE}}(f, z)=\frac{z}{2 \sqrt{\frac{\pi \cdot \lambda}{c \cdot \rho} \cdot f}},
\end{gathered}
$$

where $t$ is the time, $f$ is the frequency of spectral component, $C_{A M P}$ is the amplitude attenuation coefficient (solid line in figure 1a), $\Delta t_{\text {PHASE }}$ is the time shift (solid line in figure $\left.1 \mathrm{~b}\right), \lambda$ is the skin heat conductivity $(\lambda \approx 0.33 \mathrm{~W} /(\mathrm{m} \cdot \mathrm{K}), c$ is the specific heat $(c=3780 \mathrm{~J} /(\mathrm{K} \cdot \mathrm{kg})), \rho$ is the skin density $\left(\rho=1057 \mathrm{~kg} / \mathrm{m}^{3}\right), z$ is the thickness of a skin layer. Since the skin is a multilayer medium and each layer describes its thermal properties, $z$ value should be considered as the effective thickness. This thickness characterizes the path length of the thermal wave taking into account its volume-averaged thermal properties.

\section{Method of transformation the temperature oscillations into skin blood flow oscillations}

To realize thermography-based imaging of blood perfusion we used three steps in each point of the thermogram: the computation of the time-frequency spectrum of temperature oscillations (step I in figure 2); the compensation of an amplitude attenuation and delay time of spectral components in accordance with dependencies in

\begin{tabular}{|c|c|c|c|}
\hline & & II & III \\
\hline $\begin{array}{l}\text { Temperature } \\
\text { oscillations } \mathbf{T}(\mathbf{t})\end{array}$ & $\begin{array}{l}\text { Computation of the } \\
\mathbf{S}_{\mathbf{T}}(\mathbf{f}, \mathbf{t})- \\
\text { components of } \mathrm{T}(\mathrm{t}) \\
\text { (direct wavelet or } \\
\text { Fourier transform) }\end{array}$ & $\begin{array}{l}\text { Modification of spectral } \\
\text { components } \mathrm{S}_{\mathrm{T}}(\mathrm{f}, \mathrm{t}) \text { : } \\
\text { amplification (Eq. (2)) } \\
\text { and time shift (Eq. (3)) }\end{array}$ & $\rightarrow \begin{array}{l}\text { Synthesis of modified components } \\
\mathrm{S}_{\mathrm{BF}}(\mathrm{f}, \mathrm{t}) \text { (inverse wavelet or } \\
\text { Fourier transform), plotting of the } \\
\text { blood flow curve } \mathrm{BF}(\mathrm{t}) \text {. }\end{array}$ \\
\hline
\end{tabular}
fig. 1 (step II in figure 2); and the inverse transformation of the modified spectrum into the signal considered as a blood perfusion (step III in figure 2).

Fig. 2. Scheme of transformation the temperature signal into the blood flow signal 


\section{Results and discussion}

To demonstrate capabilities of thermography-based blood perfusion imaging under rest condition and during reaction on brachial cuff occlusion we used the following protocol: 10 minutes of simultaneously recordings of the temperature and photoplethysmography signal under rest condition, followed by 2 minutes of total brachial occlusion, succeeded by observation of hyperemia and ended by a state of rest. Special software TBF-Converter was developed to realized transformation (see figure 2) temperature into the blood flow signal, and vice versa.

\subsection{Blood flow extraction during brachial cuff occlusion}

Figure 3a shows simultaneously recorded temperature and photoplethysmography data during test with brachial cuff occlusion. It is clearly seen differences in waveforms of the two signals and a slow decrease in temperature with simultaneously abrupt drop in blood flow within occlusion period from 10 to 12 minutes.

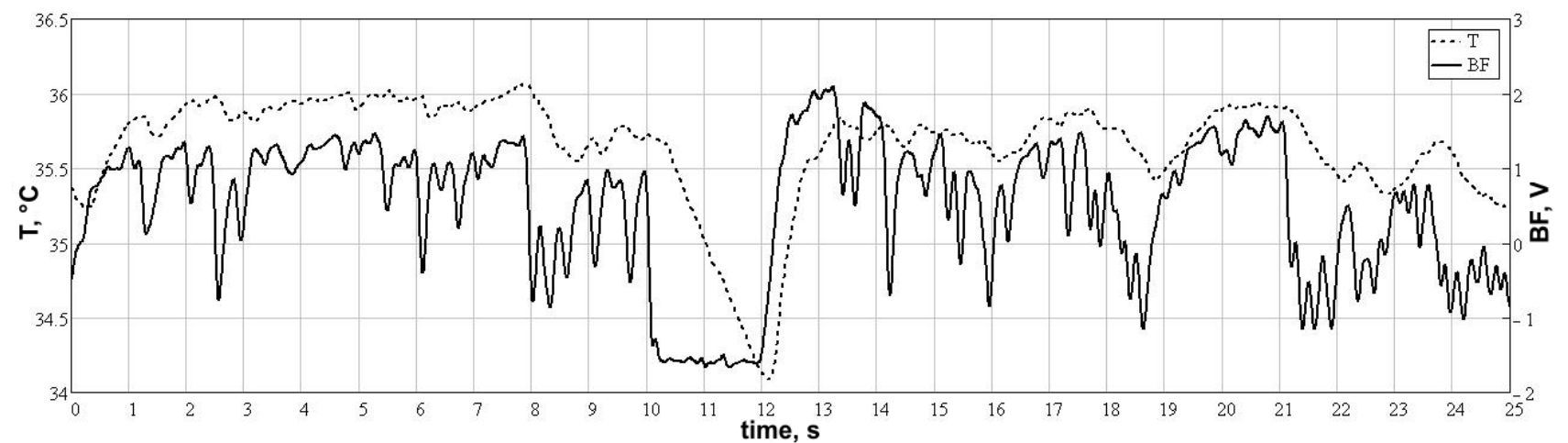

(a)

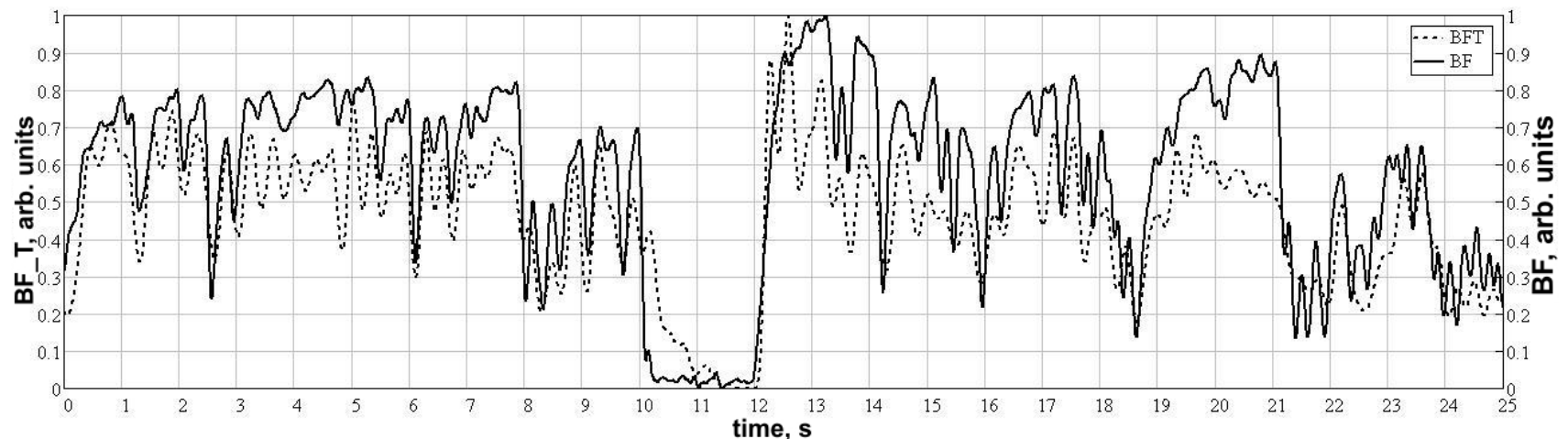

(b)

Fig 3. Time dependencies of simultaneously measured temperature (dashed line) and blood flow (solid line) oscillations from index finger (a), normalized blood flow oscillations (solid line) and blood flow extracted from temperature data (dashed line) (b)

Figure $3 \mathrm{~b}$ demonstrates the blood flow signal extracted from temperature data by means of modification of temperature spectral components in accordance with the diagram in figure 2. Comparison of the curves in figure $3 \mathrm{~b}$ makes it evident that the waveforms of blood flow oscillations are very similar, and verify a good result of the blood flow extraction. Correlation coefficients between photoplethysmography-measured blood flow and blood flow extracted from temperature, as a rule, had values above 0.75 .

The Morlet wavelet transform and blood flow extraction were evaluated in each pixel of the IR- thermogram to visualized the distribution of blood perfusion in skin of hands (see figure 4). 


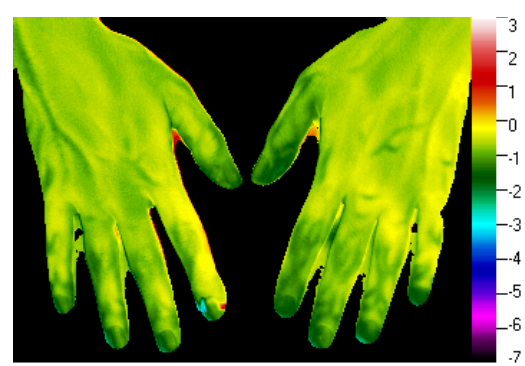

$2 \min$

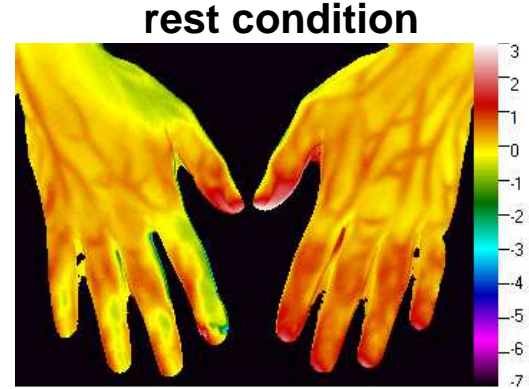

$4 \mathrm{~min}$

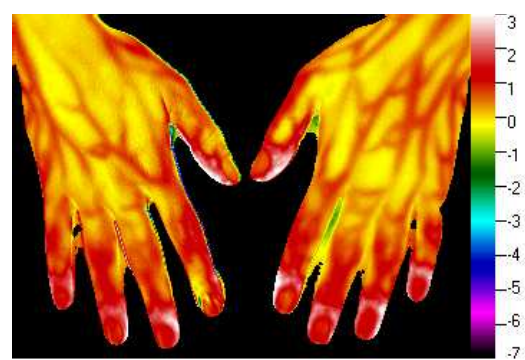

$8 \mathrm{~min}$

\section{brachial occlusion (right hand)}

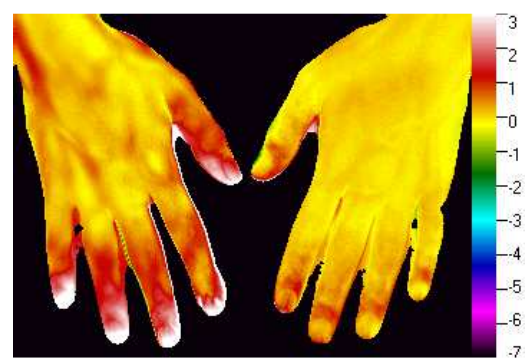

$10 \min$

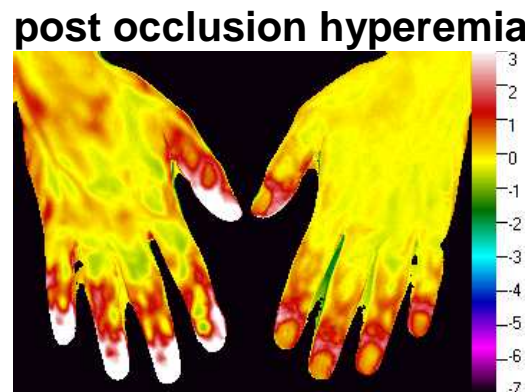

$13 \mathrm{~min}$

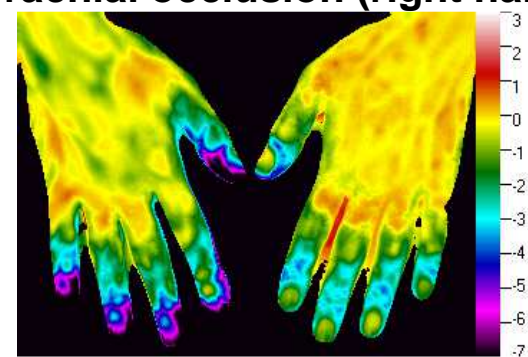

$11 \mathrm{~min}$

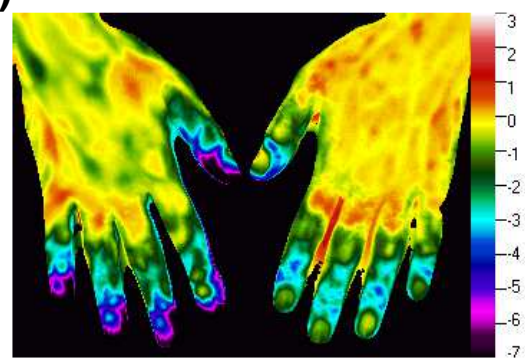

$12 \mathrm{~min}$

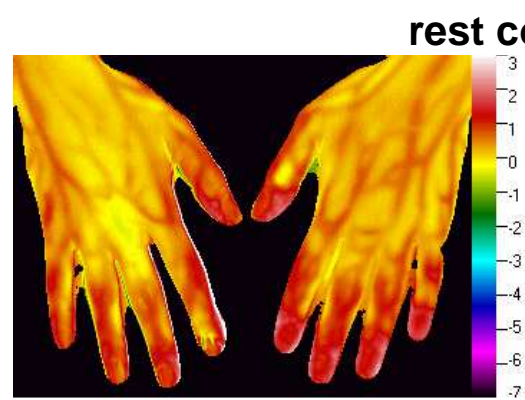

$18 \mathrm{~min}$

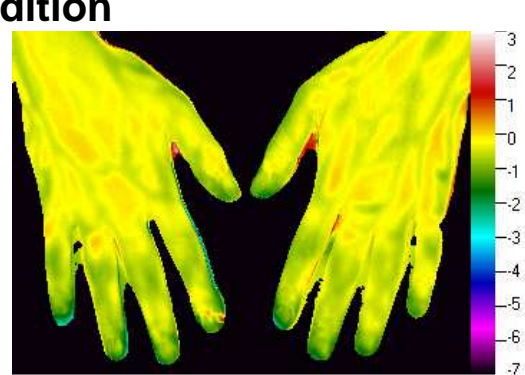

$22 \min$

Fig 4. Thermography-based perfusion imaging at the back of hands during a rest, brachial occlusion, and hyperemia conditions. (The corresponding videos of perfusion map will be available on: www.LiveTIR.com from July 1, 2014)

Figure 4 shows uniformly and symmetrical distribution of skin blood perfusion of the right and left hand during a rest condition. It is also evident variation in perfusion from low $(2 \mathrm{~min})$ to high $(8 \mathrm{~min})$ values. The brachial occlusion of right hand leads to short increasing of perfusion (10 min, fingertips of right hand) followed by abrupt decreasing of perfusion (11, $12 \mathrm{~min})$. Post occlusion hyperemia accompanied by asymmetrical increasing of perfusion of right hand, especially in fingertips. Then special distribution of blood perfusion becomes symmetric in the right and left hands (18, 22 $\min )$. 


\subsection{Software "Temperature into Blood Flow Converter" (TBF-Converter).}

To simplify the process of transformation one signal into another we have developed multifunctional program TBF-Converter 1.0 (figure 5).

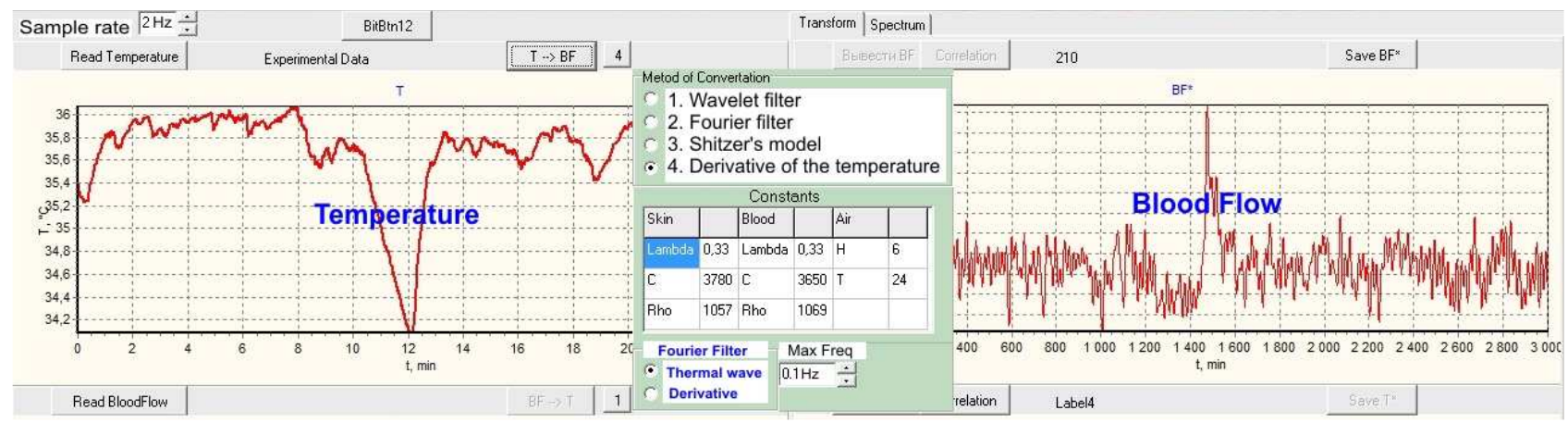

Fig 5. Screenshot of the program TBF-Converter 1.0 developed and applied for transformation of skin temperature into the blood flow signal, and vice versa

Main features of TBF-Converter $\mathbf{1 . 0}$ are the following:

1. Reading and saving the data.

2. Extraction of the blood flow signal from the photoplethysmography signal by evaluating the envelope of peaks.

3. Selection of the direction and method of transformation (wavelet transform, Fourier transform, Shitzer's model).

3.1 Transformation of temperature signal into blood flow signal.

3.2 Transformation of blood flow signal into temperature signal.

4. Definition of properties for a skin, blood and air.

5. Scaling of the curves (normalization or linear scaling).

6. Computation of the correlation coefficient between the curves.

The program TBF-Converter 1.0 will be available for download on www.LiveTIR.com from July 1, 2014.

\section{Conclusion}

Relationship between the skin temperature and skin blood flow is a central issue for the development of thermal imaging methods of skin blood flow visualization. In this study, we used the hypothesis according to which skin temperature oscillations are the result of thermal waves generated by blood flow variation. This approach allowed us establishing the laws of amplitude attenuation (Eq. (2)) and phase shift (Eq. (3)) in skin temperature as a function of its frequency (figure $1 \mathrm{a}$ and $\mathrm{b})[8]$.

The compensation of an amplitude attenuation and delay time of temperature spectral components by means of Eq. (1) gave possibility of extraction blood flow signal. Application of algorithm in figure 2 in the each pixel of the thermogram allowed us to visualize the distribution of blood flow oscillations at the back of hands during three different physiological conditions (figure 4). Proposed method of thermography-based imaging of blood perfusion has high special and time resolution, and the method is independent from any light source characteristics which are meaningful for laser Doppler or speckle contrast imaging.

The developed program TBF-Converter 1.0 simplify the process of converting the temperature into blood flow signal, and vice versa. It would be interesting, converting the Laser Doppler flowmetry signal into the skin temperature in a perspective. Thus, present study has been demonstrated the method of thermography-based blood perfusion imaging which can be promising, especially in a monitoring of skin engraftment and researches of burn injuries.

\section{REFERENCES}

[1] Jiang S.C., Ma N., Li H.J. and Zhang X.X., "Effects of thermal properties and geometrical dimensions on skin burn injuries". Burns, vol. 28, pp. 713-717, 2002.

[2] Liu J., Chen X. and Xu L.X., "New thermal wave aspects on burn evaluation of skin subjected to instantaneous heating". IEEE transactions on biomedical engineering, vol. 46, pp. 420-428, 1999.

[3] Sagaidachnyi A. A., Usanov D. A., Skripal A. V. and Fomin A. V. "Restoration of finger blood flow oscillations by means of thermal imaging". 11-th Int. Conf. on Quantitative InfraRed Thermography (QIRT 2012) (Naples, Italy) (http://qirt.gel.ulaval.ca/archives/qirt2012/papers/QIRT-2012-115.pdf).

[4] Sagaidachnyi A. A., Usanov D. A., Skripal A. V. and Fomin A. V. "Correlation of skin temperature and blood flow oscillations". Proc. SPIE 8337, Saratov Fall Meeting 2011: Optical Technologies in Biophysics and Medicine XIII, pp 83370A-8, 2012.

[5] Burton A. C., Taylor R. M., "A study of the adjustment of peripheral vascular tone to the requirement of the regulation of body temperature". Am. J. Physiol., vol. 129, pp. 566-577, 1940. 
[6] Shitzer A., Stroschein L. A., Vital P., Gonzalez R. R. and Pandolf K. B. "Numerical analysis of an extremity in a cold environment including countercurrent arterio-venous heat exchange". Journal of biomechanical engineering. vol.119, №2, pp.179-86, 1997.

[7] Ninet J. and Fronek A. "Cutaneous postocclusive reactive hyperemia monitored by laser doppler flux metering and skin temperature". Microvascular research. vol. 30, pp.125-32,1985.

[8] Sagaidachnyi A. A., Skripal A. V., Fomin A. V. and Usanov D. A. "Determination of the amplitude and phase relationships between oscillations in skin temperature and photoplethysmography-measured blood flow in fingertips". Physiological measurement, vol. 35(2), p. 153, 2014. 